\title{
PRIMITIVE NONCOMMUTATIVE JORDAN ALGEBRAS WITH NONZERO SOCLE
}

\author{
ANTONIO FERNANDEZ LOPEZ AND ANGEL RODRIGUEZ PALACIOS
}

\begin{abstract}
Let $A$ be a nondegenerate noncommutative Jordan algebra over a field $K$ of characteristic $\neq 2$. Defining the socle $S(A)$ of $A$ to be the socle of the plus algebra $A^{+}$, we prove that $S(A)$ is an ideal of $A$; then we prove that if $A$ has nonzero socle, $A$ is prime if and only if it is primitive, extending a result of Osborn and Racine [6] for the associative case. We also describe the prime noncommutative Jordan algebras with nonzero socle and in particular the simple noncommutative Jordan algebras containing a completely primitive idempotent. In fact we prove that a nondegenerate prime noncommutative Jordan algebra with nonzero socle is either (i) a noncommutative Jordan division algebra, (ii) a simple flexible quadratic algebra over an extension of the base field, (iii) a nondegenerate prime (commutative) Jordan algebra with nonzero socle, or (iv) a $K$-subalgebra of $L_{W}(V)^{(\lambda)}$ containing $F_{W}(V)$ or of $H\left(L_{V}(V),{ }^{*}\right)^{(\lambda)}$ containing $H\left(F_{V}(V),{ }^{*}\right)$ where in the first case $(V, W)$ is a pair of dual vector spaces over an associative division $K$-algebra $D$ and $\lambda \neq 1 / 2$ is a central element of $D$, and where in the second case $V$ is self-dual with respect to an hermitian inner product (|), $D$ has an involution $\alpha \rightarrow \bar{\alpha}$ and $\lambda \neq 1 / 2$ is a central element of $D$ with $\lambda+\bar{\lambda}=1$.
\end{abstract}

1. Introduction. Our aim in this paper is to obtain for noncommutative Jordan algebras an analog of the theory of primitive associative rings with minimal one-sided ideals. In [6] Osborn and Racine have solved this problem for the class of prime Jordan algebras but, as we will show, this class coincides with that of primitive Jordan algebras, in the sense of Hogben and McCrimmon [2], under the hypothesis of nonzero socle. Next we will recall these precedings of our work in order to fix notation.

All the algebras we consider here are over a field of characteristic not 2. A nonassociative algebra $A$ satisfying:

(i) $(x, y, x)=0, x, y \in A$ (Flexible Law) where $(x, y, z)=(x y) z-x(y z)$ is the associator of $x, y, z$,

(ii) $\left(x^{2}, y, x\right)=0, x, y \in A$ (Jordan identity), is called a noncommutative (in short n.c.) Jordan algebra. Let $A$ be a n.c. Jordan algebra. For any $\mu \in K$ we can define a new algebra structure on $A$, the $\mu$-mutation $A^{(\mu)}$, by $x \cdot{ }_{\mu} y=\mu x y+(1-\mu) y x$. As usual $A^{(1 / 2)}$ is denoted by $A^{+}$. We recall that $A^{+}$is a Jordan algebra whose product is denoted by $x \cdot y$. An algebra of the form $A=B^{(\mu)}$ for $B$ associative is called a split quasiassociative algebra. An algebra is quasiassociative when it has a scalar extension $A_{F}$ which is split quasiassociative. (We can always choose a quadratic extension [1, p. 583].) We recall that if $J$ is a nondegenerate Jordan algebra, $U_{a}=0$ implies $a=0$, then the socle $S(J)$ of $J$ is

Received by the editors January 15, 1985 and, in revised form, April 2, 1985.

1980 Mathematics Subject Classification. Primary 17C10; Secondary 17C20, 16A68. 
defined to be the span of all minimal inner ideals of $J$ [6]. We also recall [5] that for any n.c. Jordan algebra there exists a unique maximal quasi-invertible ideal $\operatorname{Rad}(A)$ called the Jacobson radical of the algebra $A$. $A$ is semisimple if $\operatorname{Rad}(A)=0$. For a n.c. Jordan algebra $A$ we define a subset $I$ to be a maximal-modular inner ideal if $I$ is a maximal-modular inner ideal of $\mathrm{A}^{+}$, and we define the core of a maximal-modular inner ideal $I$ as the largest ideal of $A$ contained in $I$. For $A$ commutative, Hogben and McCrimmon [2] have characterized the radical of $A$ as the intersection of the cores of the maximal-modular inner ideals of $A$. This result together with the fact that the Jacobson radical of a n.c. Jordan algebra $A$ is the largest ideal of $A$ contained in $\operatorname{Rad}\left(A^{+}\right)[5$, Theorem 11] yields the following theorem.

THEOREM 1. The Jacobson radical of a n.c. Jordan algebra is the intersection of the cores of its maximal-modular inner ideals.

Following [3, p. 69] let $(V, W)$ denote a pair of dual vector spaces over a division ring $D$. We recall that an element $a \in \operatorname{Hom}_{D}(V, V)$ is said to be continuous if there exists $a^{*} \in \operatorname{Hom}_{D}(W, W)$, necessarily unique, such that $(v a, w)=\left(v, a^{*} w\right)$ for all $v \in V, w \in W . L_{W}(V)$ is the ring of continuous linear transformations of $V$. $F_{W}(V)$ is the ideal of all elements with finite rank.

The subrings of $L_{W}(V)$ containing $F_{W}(V)$ are characterized as those primitive rings having nonzero socle. If such a ring has an involution *, then $D$ has an involution, $V$ is self-dual with respect to an hermitian or symplectic inner product (|) and the involution * is the adjoint with respect to (|). We remark that in the symplectic case $D$ is a field and its involution is the identity. Also one can see that such rings are algebras over $K$ when $D$ is a $K$-algebra.

Let $V$ be a self-dual vector space and let $H\left(L_{V}(V),{ }^{*}\right)$ denote the Jordan algebra of all symmetric elements of $\left(L_{V}(V),{ }^{*}\right)$. Osborn and Racine [6] have shown that the Jordan subalgebras of $H\left(L_{V}(V),{ }^{*}\right)$ containing $H\left(F_{V}(V),{ }^{*}\right)$ or of $L_{W}(V)^{+}$containing $F_{W}(V)$ together with the simple Jordan algebras with descending chain condition on principal inner ideals are the only nondegenerate prime Jordan algebras with nonzero socle.

Now let $A$ be a $K$-subalgebra of $L_{W}(V)^{(\lambda)}$ containing $F_{W}(V)$, where the division ring $D$ is an algebra over $K$ and $\lambda$ is a central element of $D$. Since $A^{+}$is then a Jordan subalgebra of $L_{W}(V)^{+}$containing $F_{W}(V)$ then we have that $A^{+}$is a nondegenerate prime Jordan algebra with socle $F_{W}(V)^{+}$. Hence $A$ is a nondegenerate prime noncommutative Jordan algebra with "socle" $F_{W}(V)^{(\lambda)}$. Similarly the $K$-subalgebras of $H\left(L_{V}(V),{ }^{*}\right)^{(\lambda)}$ containing $H\left(F_{V}(V),{ }^{*}\right)$, where $\lambda$ is a central element of $D$ with $\lambda+\bar{\lambda}=1$ are nondegenerate prime noncommutative Jordan algebras with socle $H\left(F_{V}(V),{ }^{*}\right)^{(\lambda)}$. We must note that symplectic inner products occur only in the commutative case. Indeed, as we have already pointed out, $\alpha \rightarrow \bar{\alpha}$ is the identity on $D$ in this case and together with $1=\lambda+\bar{\lambda}$ implies $\lambda=1 / 2$.

Other noncommutative Jordan algebras with these properties are the simple noncommutative Jordan algebras with descending chain condition on inner ideals described by McCrimmon in [5]. 
We prove in this work that the noncommutative Jordan algebras cited above are the only nondegenerate prime noncommutative Jordan algebras with nonzero socle, where the socle, the span of all minimal inner ideals, is shown to be an ideal.

2. Simple noncommutative Jordan algebras containing a completely primitive idempotent. For any element $a$ in a n.c. Jordan algebra $A, U_{a}$ denotes the linear operator on $A$ defined by $U_{a} x=a(a x+x a)-a^{2} x=(a x+x a) a-x a^{2}, x \in A$. We recall that $U_{a}=U_{a}^{+}$where $U_{a}^{+}$is the usual $U$-operator on the Jordan algebra $A^{+}$. An idempotent $e$ in $A$ is completely primitive if the Peirce 1-space $A_{1}(e)=U_{e} A$ is a division algebra. A unital n.c. Jordan algebra has capacity if the unity is a sum of mutually orthogonal completely primitive idempotents.

Lemma 2. Let $J$ be a Jordan $K$-algebra and let $F$ be a quadratic extension of $K$.

(i) If $J$ is semisimple then the scalar extension $J_{F}$ is also semisimple.

(ii) If $J$ is a division algebra then $J_{F}$ has capacity.

Proof. (i) Without loss in generality we can assume $F=K \oplus \alpha K$ where $\alpha$ is an element of $F$ which is not in $K$ with $\alpha^{2} \in K$, and $J_{F}=J \oplus \alpha J$. Let ${ }^{*}$ be the canonical involution $a+\alpha b \rightarrow a-\alpha b$ of $J_{F}$ which is conjugate linear with respect to the automorphism $\varepsilon_{1}+\alpha \varepsilon_{2} \rightarrow \varepsilon_{1}-\alpha \varepsilon_{2}$ of $F$. Since $\operatorname{Rad}\left(J_{F}\right)$ is a selfadjoint ideal we have that $\operatorname{Rad}\left(J_{F}\right) \cap J \neq 0$ whenever $\operatorname{Rad}\left(J_{F}\right) \neq 0 . \operatorname{But} \operatorname{Rad}\left(J_{F}\right) \cap J$ is contained in $\operatorname{Rad}(J)$ by uniqueness of quasi-inverse. Thus $\operatorname{Rad}\left(J_{F}\right)=0$ because $J$ is semisimple.

(ii) By [10] either $J=D^{+}$where $D$ is an associative division algebra or $J=$ $H\left(D,{ }^{*}\right)$, the symmetric elements of an associative division algebra $D$ with an involution *, or $J=K 1 \oplus X$, the Jordan algebra of a nondegenerate symmetric bilinear form on a vector space $X$, or $J$ is an exceptional Jordan division algebra which is finite dimensional over its centre.

In the first case $D_{F}=D \oplus \alpha D$ is an associative $F$-algebra with $J_{F}=D_{F}^{+} . D_{F}$ is semisimple and it can be regarded as a left vector space over $D$ of dimension one or two. Hence it is easy to conclude that $J_{F}$ has capacity one or two. Now let us suppose that $J=H\left(D,{ }^{*}\right)$. As above we consider the algebra $D_{F}$ and extend the involution of $D$ to $D_{F}$ defining $(a+\alpha b)^{*}=a^{*}+\alpha b^{*}$. The foregoing argument shows that either $\left(D_{F},{ }^{*}\right)$ is ${ }^{*}$-simple containing a minimal one-sided ideal or is a direct sum of two such ideals. But $J_{F}=H\left(D_{F},{ }^{*}\right)$, so that, by [6, Theorem 9], $J_{F}$ has capacity one or two. Now we consider the case when $J=K 1 \oplus X$. Let $X_{F}=X \oplus \alpha X$ be the scalar extension of $X$ and let (|) be the unique bilinear form which extends that of $X$ to $X_{F}$. It is easy to see that $J_{F}=F 1 \oplus X_{F}$. Therefore $J_{F}$ has capacity one or two. We must consider finally the case when $J$ is a finite-dimensional algebra over its centre $Z$. By (i) $J_{F}$ is semisimple and since every principal inner ideal of $J_{F}$ is invariant under the multiplication by elements of $Z$, we have by $[4$, Theorem $3, \mathrm{p}$. 158] that $J_{F}$ has capacity.

COROllaRY 3. Let $A$ be a n.c. Jordan $K$-algebra containing a completely primitive idempotent and let $F$ be a quadratic extension of $K$. Then the scalar extension $A_{F}$ contains a completely primitive idempotent. 
Proof. Let $e$ be a completely primitive idempotent of $A$. Clearly $U_{e} A_{F}$ is the scalar extension of $U_{e} A$. Since the inverses in $U_{e} A$ and $\left(U_{e} A\right)^{+}$are the same [5, p. 4], Lemma 2 applied to the Jordan division algebra $\left(U_{e} A\right)^{+}$shows that $U_{e} A_{F}$ contains a completely primitive idempotent, which remains such as in $A_{F}$, as is well known.

THEOREM 4. A n.c. Jordan K-algebra is simple containing a completely primitive idempotent if and only if it is one of the following:

(i) A n.c. Jordan division algebra.

(ii) A simple flexible quadratic algebra over an extension of the base field.

(iii) A simple (commutative) Jordan algebra containing a completely primitive idempotent.

(iv) A quasiassociative algebra of the form $F_{W}(V)^{(\lambda)}$ or $H\left(F_{V}(V),{ }^{*}\right)^{(\lambda)}$ where in the first case $(V, W)$ is a pair of dual vector spaces over a division $K$-algebra $D$ and $\lambda \neq 1 / 2$ is a central element of $D$, and where in the second case $V$ is self-dual with respect to an hermitian inner product, $D$ has an involution $\alpha \rightarrow \bar{\alpha}$ and $\lambda \neq 1 / 2$ is $a$ central element of $D$ with $\lambda+\bar{\lambda}=1$.

Proof. Suppose that $A$ is a simple n.c. Jordan $K$-algebra containing a completely primitive idempotent $e$. If $e$ is a unity for $A$, then $A$ is a division algebra and we are done: $A$ is as in (i). Otherwise we have by [5, Theorem 1] that $A^{+}$is a simple Jordan algebra containing a completely primitive idempotent. Then by [6, Corollary 7] either $A^{+}$, and therefore $A$ also, is unital with (finite) capacity or it contains a subalgebra of capacity $n$ for any positive integer $n$. The case that $A$ is unital with capacity one has just been rejected. If $A$ is unital with capacity two, then by [9] applied to the algebra $A$, regarded as a simple algebra over its centroid $C$, either $A$ is as in (ii) or (iii), or $A$ is quasiassociative over $C$. In the remainder cases by [5, Theorem 5] either $A$ is as in (iii) or $A$ is quasiassociative over $C$. Thus we only need to consider the case when $A$ is quasiassociative over $C$. If $A$, as an algebra over $C$, is split quasiassociative, then $A=B^{(\lambda)}$ with $B$ associative and $\lambda \in C, \quad \lambda \neq 1 / 2$. Clearly $B$ is simple with minimal one-sided ideals. Therefore there exists a pair of dual vector spaces $(V, W)$ over a division $K$-algebra $D$ such that $A=F_{W}(V)^{(\lambda)}$ with $\lambda$ in the centre of $D$ and $\lambda \neq 1 / 2$. Otherwise there exists a quadratic extension $F$ of $C$ such that $A_{F}=B^{(\lambda)}$ where $B$ is an associative $F$-algebra and $\lambda \in F$, $\lambda \neq 1 / 2$. Since $A$, as an algebra over $C$, is central simple we have by [4, p. 206] that $A_{F}$ is simple. Moreover $A_{F}$ contains a completely primitive idempotent by Corollary 3. Hence $B$ is a simple associative algebra containing minimal one-sided ideals. Now $\lambda(1-\lambda)=\gamma$ with $\gamma \in C[1$, Theorem 5, p. 583], and hence $\lambda=1 / 2+\alpha$ where $\alpha$ is an element of $F$ which is not in $C$ but $\alpha^{2} \in C$. Let $\beta \rightarrow \bar{\beta}$ denote the canonical automorphism $\varepsilon_{1}+\alpha \varepsilon_{2} \rightarrow \varepsilon_{1}-\alpha \varepsilon_{2}$ of $F=C \oplus \alpha C$ and let $x \rightarrow x^{*}$ be the mapping of $A_{F}=A \oplus \alpha A$ into itself defined by $(a+\alpha b)^{*}=a-\alpha b$, which satisfies $(\beta x)^{*}$ $=\bar{\beta} x^{*},\left(x_{1}+x_{2}\right)^{*}=x_{1}^{*}+x_{2}^{*},\left(x_{1} x_{2}\right)^{*}=x_{1}^{*} x_{2}^{*}$ and $\left(x^{*}\right)^{*}=x$ for all $x, x_{1}, x_{2}$ in $A_{F}, \beta \in F$. Clearly $\lambda+\bar{\lambda}=1$ and if $\mu=\lambda /(2 \lambda-1)$ we have that $\mu+\bar{\mu}=1$ also. But $B=A_{F}^{(\mu)}\left[5\right.$, p. 18], so that ${ }^{*}$ is a (linear) involution on $B$, regarded as an algebra over $C$. Also it is clear that $A=H\left(B,{ }^{*}\right)^{(\lambda)}$. Therefore $A=H\left(F_{V}(V),{ }^{*}\right)^{(\lambda)}$ where $V$ is self-dual with respect to an hermitian inner product $(\mid)$ with respect to a 
division $K$-algebra with involution $(D,-)$ and $\lambda \neq 1 / 2$ is a central element of $D$ with $\lambda+\bar{\lambda}=1$. We recall that symplectic inner products can not occur here as we have remarked in the introduction.

The converse follows from [6, Theorem 9] taking into account that $B$ and $B^{(\lambda)}$ have the same Jordan structure for any associative algebra $B$.

3. The socle of a nondegenerate noncommutative Jordan algebra. Let $A$ be a nondegenerate n.c. Jordan algebra. We define the socle $S(A)$ of $A$ to be the span of the minimal inner ideals of $A$; as usual $S(A)=0$ if $A$ does not contain any minimal inner ideal. It is clear that $S(A)=S\left(A^{+}\right)$. Thus by [6, Theorem 17] $S(A)$ is an ideal of $A^{+}$. Next we shall prove that $S(A)$ is actually an ideal of $A$. Let $A$ be a nonassociative algebra. By a derivation of $A$ is meant a linear operator $D$ on $A$ satisfying $D(x y)=x D(y)+D(x) y$ for all $x, y \in A$. If $M$ is an ideal of $A$, it is not difficult to see that both $M+D(M)$ and $M \cap D^{-1}(M)$ are ideals of $A$.

Lemma 5. If $M$ is a minimal ideal of $A$ with $M^{2} \neq 0$ then $D(M) \subset M$ for any derivation $D$ of $A$.

Proof. $M \cap D^{-1}(M)$ is a nonzero ideal of $A$. Indeed, let $x, y \in M$ such that $x y \neq 0$. Then $D(x y)=x D(y)+D(x) y \in M$, so that $0 \neq x y \in M \cap D^{-1}(M)$. Hence $M=M \cap D^{-1}(M)$ by minimality of $M$, so that $D(M) \subset M$.

Suppose now that $A$ is flexible. It is well known [7, p. 146] that the mapping $x \rightarrow[a, x]=a x-x a$ is a derivation of the plus algebra $A^{+}$.

Proposition 6. Suppose that $A$ is flexible and let $M$ be a simple ideal of the plus algebra $A^{+}$. Then $M$ is a simple ideal of $A$.

Proof. By Lemma $5[a, x] \in M$ for all $a \in A, x \in M$. Hence it is clear that $M$ is an ideal, plain simple, of $A$.

THEOREM 7. The socle of a nondegenerate n.c. Jordan algebra $A$ is the direct sum of simple ideals containing a completely primitive idempotent.

Proof. Let $M$ be a simple ideal of $A^{+}$containing a completely primitive idempotent (see [6, Lemma 15]). By Proposition 6, $M$ is a simple ideal of $A$. Thus the proof is now a direct consequence of [6, Theorem 17].

Now let $A$ be a semiprime associative algebra. An idempotent $e \neq 0$ is completely primitive in the Jordan algebra $A^{+}$iff $e A$ is a minimal right ideal of $A$. Then it is not difficult to see that the notion of socle for a n.c. Jordan algebra agrees with that usual for an associative algebra. On the other hand this definition coincides also with that of Slater [8] for the alternative case.

COROllary 8. The socle of a nondegenerate alternative algebra $A$ coincides with the socle of $A$ as an alternative algebra.

Proof. Since by [8, Theorem 3] any minimal ideal of $A$ is either nuclear or a Cayley-Dickson algebra over its centre, the proof can be reduced to the associative case. 
4. Primitive noncommutative Jordan algebras with nonzero socle. Let $A$ be a n.c. Jordan algebra. An ideal $P$ of $A$ is said to be prime when $I_{1} I_{2} \subset P\left(I_{1}\right.$ and $I_{2}$ ideals of $A$ ) implies $I_{1} \subset P$ or $I_{2} \subset P$. $A$ is said to be prime when 0 is a prime ideal. We also recall that $A$ is called primitive if it contains a maximal-modular inner ideal with zero core. By making use of the methods of [2, Proposition 5.5] one can prove that every primitive n.c. Jordan algebra is prime. Next we shall settle the converse under the hypothesis of nonzero socle.

For any ideal $I$ of a nonassociative algebra $A$ we define the annihilator of $I$, Ann $(I)$ as the largest ideal $B$ of $A$ satisfying $I B=B I=0 . A^{+}(I)$ stands for the annihilator of $I$ in the plus algebra $A^{+}$.

Lemma 9. Suppose that $A$ is flexible and let I be an ideal of $A$. Then $\operatorname{Ann}^{+}(I)$ is an ideal of $A$.

Proof. Let $D_{a}$ denote the derivation $x \rightarrow[a, x]$ of $A^{+}$. Then, as it was already pointed out, $D_{a}\left(\operatorname{Ann}^{+}(I)\right)+\operatorname{Ann}^{+}(I)$ is an ideal of $A^{+}$. Moreover $D_{a}(x) \cdot y=$ $D_{a}(x \cdot y)-x \cdot D_{a}(y)=0$ for all $x \in \operatorname{Ann}^{+}(I), y \in I$ because $D_{a}(I) \subset I$. Thus $\left[A, \operatorname{Ann}^{+}(I)\right] \subset \operatorname{Ann}^{+}(I)$. Hence it follows that $\operatorname{Ann}^{+}(I)$ is an ideal of $A$.

Corollary 10. Let $A$ be a flexible algebra such that $B^{2} \neq 0$ for any nonzero ideal $B$ of $A$. Then $\operatorname{Ann}^{+}(I)=\operatorname{Ann}(I)$ for all nonzero ideals $I$ of $A$.

Proof. Clearly Ann $(I) \subset \mathrm{Ann}^{+}(I)$. Now by Lemma $9 \mathrm{Ann}^{+}(I)$ is an ideal of $A$ satisfying $\left(I \cap \operatorname{Ann}^{+}(I)\right)^{2} \subset I \cdot \operatorname{Ann}^{+}(I)=0$. Thus $I \cap \operatorname{Ann}^{+}(I)=0$ by hypothesis. Then $\operatorname{Ann}^{+}(I) \subset \operatorname{Ann}(I)$.

Proposition 11. Let $A$ be a n.c. Jordan algebra. If $M$ is a minimal ideal of $A$ containing a nonzero idempotent, then $\operatorname{Ann}(M)$ is the core of a maximal-modular inner ideal of $A$.

Proof. Let $e$ be a nonzero idempotent of $A$. Since $U_{e} A \subset M$ we have that $\operatorname{Ann}(M)$ is contained in $U_{1-e}(A)$, which is a proper $e$-modular inner ideal [2,2.8], so that it is contained in an $e$-maximal inner ideal $I$ of $A$. Now let $B$ be an ideal of $A$ contained in $I$. If $B$ is not contained in $\operatorname{Ann}(M)$, then we have by minimality of $M$ that $M \subset B$. Hence $e \in I$, which is a contradiction since $I$ is proper [2, Proposition 3.1]. Therefore $\operatorname{Ann}(M)$ is the largest ideal of $A$ contained in $I$, that is, $\operatorname{Ann}(M)$ is the core of $I$.

THEOREM 12. Let $A$ be a nondegenerate n.c. Jordan algebra with nonzero socle. Then the following conditions are equivalent:

(i) $\mathrm{A}^{+}$is a primitive Jordan algebra;

(ii) $A$ is primitive;

(iii) $A$ is prime.

Proof. Clearly (i) implies (ii), and (ii) implies (iii) as we have already pointed out at the first of this section. Thus we must prove only that (iii) implies (i). By primeness $S(A)=S\left(A^{+}\right)=M$ is a simple ideal of $A$. But since $A$ is nondegenerate it follows from Corollary 10 that $\operatorname{Ann}^{+}(M)=0=\operatorname{Ann}(M)$ by primeness again. Thus $A^{+}$is primitive by Proposition 11 applied to the Jordan algebra $A^{+}$. 
RemarK. The equivalence (ii) $\Leftrightarrow$ (iii) was settled by Osborn and Racine in [6, Theorem 1] for an associative algebra $A$.

THEOREM 13. A n.c. Jordan $K$-algebra $A$ is nondegenerate prime with nonzero socle if and only if it is one of the following:

I. A n.c. Jordan division K-algebra;

II. A simple flexible quadratic algebra over an extension of the base field;

III. A nondegenerate prime (commutative) Jordan K-algebra with nonzero socle;

IV. A K-subalgebra of $L_{W}(V)^{(\lambda)}$ containing $F_{W}(V)$ or of $H\left(L_{V}(V),{ }^{*}\right)^{(\lambda)}$ containing $H\left(F_{V}(V),{ }^{*}\right)$ where in the first case $(V, W)$ is a pair of dual vector spaces over a division $K$-algebra $D$ and $\lambda \neq 1 / 2$ is a central element of $D$, and where in the second case $V$ is self-dual with respect to an hermitian inner product $(\mid), D$ has an involution $\alpha \rightarrow \bar{\alpha}$ and $\lambda \neq 1 / 2$ is a central element of $D$ with $\lambda+\bar{\lambda}=1$.

Proof. Suppose first that $A$ is a nondegenerate prime n.c. Jordan $K$-algebra with socle $M \neq 0$. By primeness $M$ is a simple ideal containing a completely primitive idempotent, so that we can apply Theorem 4 to the algebra $M$. If $M$ is unital one proves as in [6, p. 384] that $A=M$ and we have finished the proof. Therefore, by virtue of Theorem 4 and the structure theorem for simple Jordan algebras containing a completely primitive idempotent [6, Theorem 9], we must consider only the cases when $M=F_{W}(V)^{(\lambda)}$ or $M=H\left(F_{V}(V),{ }^{*}\right)^{(\lambda)}$ where $\lambda$ is a central element of $D$ and $\lambda+\bar{\lambda}=1$ in the latter case. (We do not exclude $\lambda=1 / 2$.)

Assume first that $M=F_{W}(V)^{(\lambda)}$. By [6, p. 384] there exists an isomorphism $x \rightarrow \tilde{x}$ from $A^{+}$into $L_{W}(V)^{+}$defined as follows:

Given $x \in A$ and $v \in V$ we choose an idempotent $e \in M$ such that $v \tilde{e}=v$. Then $v \tilde{x}=v\left(\tilde{x}_{1}+\tilde{x}_{01}\right), x_{1}, x_{01}$ the Peirce components of $x$ with respect to $e$. This definition is independent of the choice of $e$ and it is always possible to suppose $v \tilde{x}=v \tilde{x}_{1}$. Now we must prove that $\widetilde{x y}=\lambda \tilde{x} \tilde{y}+(1-\lambda) \tilde{y} \tilde{x}$ for all $x, y \in A$. But this can be obtained as in the Jordan case $\lambda=1 / 2$ [6, p. 385] with minor alterations, so that we omit the details. We remark that if $a, b \in L_{W}(V)$ then $\lambda(a b)+(1-\lambda) b a$ $\in L_{W}(V)$ since $\lambda$ is a central element of $D$.

Analogously it is proved that there exists an isomorphism from $A$ into $H\left(L_{V}(V),{ }^{*}\right)^{(\lambda)}$ when $M=H\left(F_{V}(V),{ }^{*}\right)^{(\lambda)}$.

The converse follows from the Jordan case [6, Theorem 18] taking into account that for any associative $K$-algebra $B$ and $\lambda \in K$ the algebras $B$ and $B^{(\lambda)}$ have the same Jordan structure and that $S(B)=S\left(B^{+}\right)=S\left(B^{(\lambda)}\right)$ whenever any one of these exists.

\section{REFERENCES}

1. A. A. Albert, Power associative rings, Trans. Amer. Math. Soc. 64 (1948), 552-593.

2. L. Hogben and K. McCrimmon, Maximal modular inner ideals and the Jacobson radical of a Jordan algebra, J. Algebra 68 (1981), 155-169.

3. N. Jacobson, Structure of rings (2nd ed.), Amer. Math. Soc. Colloq. Publ., vol. 37, Amer. Math. Soc., Providence, R. I., 1964.

4. Structure and representations of Jordan algebras, Amer. Math. Soc. Colloq. Publ., vol. 39. Amer. Math. Soc., Providence, R. I., 1968. 
5. K. McCrimmon, Noncommutative Jordan rings, Trans. Amer. Math. Soc. 158 (1971), 1-33.

6. J. M. Osborn and M. L. Racine, Jordan rings with nonzero socle, Trans. Amer. Math. Soc. 251 (1979), 375-387.

7. R. D. Schafer, An introduction to nonassociative algebras, Academic Press, New York, 1966.

8. M. Slater, The socle of an alternative ring, J. Algebra 14 (1970), 443-463.

9. K. C. Smith, Noncommutative Jordan algebras of capacity two, Trans. Amer. Math. Soc. 158 (1971), 151-159.

10. E. I. Zel'manov, Jordan division algebras, Algebra i Logika 18 (1979), 286-310.

Departamento de Algebra y Fundamentos, Facultad de Ciencias, Universidad de Malaga, Malaga, Spain

Departamento de Teoria de funciones, facultad de Ciencias, Universidad de Granada, Granada, SPain 